\title{
Structure and stability of hydrogenated carbon atom vacancies in graphene
}

\author{
Marina Casartelli ${ }^{a}$, Simone Casolo ${ }^{a}$, Gian Franco Tantardini ${ }^{a, b}$ and Rocco Martinazzo ${ }^{a, b \text { F }}$ \\ a Dipartimento di Chimica, Università degli Studi di Milano, via Golgi 19, 20133 Milan, Italy. \\ ${ }^{b}$ Istituto di Scienza e Tecnologia Molecolari, Consiglio Nazionale delle Ricerche, via Golgi 19, 20133 Milan, Italy.
}

\begin{abstract}
Adsorption of hydrogen atoms to a carbon atom vacancy in graphene is investigated by means of periodic first principles calculations, up to the fully hydrogenated state where six $\mathrm{H}$ atoms chemically bind to the vacancy. Addition of a single $\mathrm{H}$ atom is highly exothermic and barrierless, and binding energies remain substantial for further hydrogenation, with a preference towards structures with the least number of geminal pairs. Thermodynamic analysis shows that defective graphene is extremely sensitive to hydrogenation, with the triply hydrogenated anti- structure prevailing at room temperature and for a wide range of $\mathrm{H}_{2}$ partial pressures, from $\sim 1$ bar down to $<10^{-20}$ bar. This structure has one unpaired electron and provides a spin-half local magnetic moment contribution to graphene paramagnetism. Comparison of our results with recent TEM, STM and $\mu$-SR experiments suggest that carbon atom vacancies may actually be hydrogenated to various degrees under varying conditions.
\end{abstract}

\section{INTRODUCTION}

Defects in graphene such as ad-species or missing carbon atoms -commonly referred to as " $p_{Z}$ vacancies"play important roles in charge transport, magnetism and chemistry of graphene. Most often they are unavoidable byproducts of the fabrication process (for instance, when reducing graphene oxide) but they can also be deliberately introduced by cold plasma treatment, heavy-ion bombardment or high-energy electrons, in the latter case employing the same transmission-electronmicroscopy (TEM) setup used to image the surface[1. Vacancies and other under-coordinated sites are known to be starting sites for metal-induced etching[2 [4], to show an enhanced chemical reactivity [5, 6] and to incorporate dopant species [7].

All the above defects introduce semi-localized $\pi$ "midgap" states which decay slowly (as $\sim 1 / r$ ) from the defect position [8 10] and host itinerant electrons which are free to move on one of the two sublattices of which graphene is made (the one opposite to the sublattice containing the closest defect). As such, they act as resonant scatterers and determine graphene conductivity at zero and finite carrier densities 11-15, provide spin-half semi-local magnetic moments detectable by magnetometry experiments 16, 17] and bias graphene chemical reactivity towards specific lattice positions 18, 19.

Carbon atom vacancies occupy a special position in this context since, in addition to the above $\pi$-"midgap" state, they have $\sigma$ dangling bonds arising upon atom removal from the breaking of the $\mathrm{C}-\mathrm{C}$ bonds which held the lattice atom in place. In the bare vacancy a structural (Jahn-Teller) distortion occurs that removes two of these bonds, as predicted by theory [20 26] and confirmed by scanning tunneling microscopy (STM) [10, 27-31] and TEM 32 experiments. Even though is not clear whether such distortion is static or dynamic 20], thereby break-

*Electronic address: rocco.martinazzo@unimi.it ing or preserving the threefold symmetry of the ideal vacancy, it leaves one single $\sigma$ electron which is free to couple with the above $\pi$ one. As a result, the ground state of a bare vacancy is a triplet, but the singlet with one spin flipped (which is only $\sim 0.2 \mathrm{eV}$ higher in energy) can be easily accessed if ripples in the graphene sheet or interaction with a substrate are taken into account 26 . However, the newly formed C-C bond is rather weak, as expected from its length $(\sim 2.0 \AA)$ which is much larger than a typical single C-C bond, and can in principle be broken by addition of e.g. $\mathrm{H}$ atoms. Thus, under typical laboratory conditions, the question arises to what extent hydrogenation occurs, and which structures result. This question is of interest in many respects: the degree of hydrogenation (and their relative arrangement) determines the residual magnetic moment of the defect, influences the structure of the carbon atom vacancy (which might be locked in a distorted geometry 32 or be statically symmetric), and possibly explain recent muon spin-resonance ( $\mu$-SR) experiments suggesting formation of geminal adducts of muonion species on singly hydrogenated vacancies.

In this paper we study the structure, the stability, the magnetic properties and the mechanism of the carbon mono-vacancy hydrogenation, from one to six hydrogen atoms, and discuss the relevance of our results to a number of experimental investigations. The paper is organized as follows: Section II outlines the method used and the adopted computational set-up, Section III discusses the results and Section IV summarizes and concludes.

\section{THEORY}

The structure and the energetics of single and multiple hydrogenated vacancies in graphene was investigated in periodic models with plane-wave density functional theory (DFT) as implemented in the Vienna ab initio simulation package (VASP) 33, 34]. The size of the supercell, as well as the relevant computational parameters (k-mesh, plane wave cutoff for expanding the wavefunc- 
tion and the charge density, etc.) were selected by preliminary tests similarly to our recent work[26] and chosen to be a good compromise between the need of minimizing interactions between periodic images and computational cost. The exchange-correlation effects were included via the Perdew-Burke-Ernzerhof [35, 36] functional within the generalized gradient approximation, in its spin-polarized form. Kohn-Sham orbitals were expanded in a plane-wave basis set limited to a $500 \mathrm{eV}$ energy cutoff and core electrons described by the projector augmented-wave 37, 38, method. A $6 \times 6$ supercell with $20 \AA$ vacuum between periodic replica normal to the surface was used, and the Brillouin zone was sampled with a $6 \times 6 \times 1, \Gamma$-centered $k$-point mesh; geometry relaxation was performed till the Hellman-Feyman forces on each atom decreased below $0.01 \mathrm{eV} / \AA$.

Reaction energies for adsorbing $n$ hydrogen atoms were computed as

$$
\Delta E_{n}=E_{V H_{n}}-E_{V}-E_{n H}
$$

where $E_{V H_{n}}$ is the total energy of the $n$-times hydrogenated vacancy (denoted as $V H_{n}$ ), $E_{V}$ that of the bare vacancy (in its ground-state) and $E_{n H}$ is the reference state of $n$ hydrogen atoms, $E_{n H}=n E_{H}$. The latter, along with the molecular energy $E_{H_{2}}$ to be introduced below, was obtained with a setup similar to that described above, using a $\Gamma$ point calculation on a cubic cell with a $20 \AA$ long side. Reaction energies for sequential adsorption, $\Delta \Delta E_{n}$, were defined similarly as

$$
\Delta \Delta E_{n}=\Delta E_{n}-\Delta E_{n-1}=E_{V H_{n}}-E_{V H_{n-1}}-E_{H}
$$

(where, unless otherwise stated, $\Delta E_{n-1}$ refers to the most stable $n$-1-times hydrogenated state) and used to compare the relative strength of the different $\mathrm{CH}$ bonds which may form during the adsorption process. We also computed a few selected activation energies (barriers) following the nudged elastic bands method [39] as implemented in VASP.

\section{RESULTS}

\section{A. Structure and energetics}

As mentioned in the introduction, a vacancy in graphene forms upon the extraction of a carbon atom which leaves three $\sigma$ dangling bonds and one unpaired $\pi$ electron on the lattice. The $\sigma$ electrons are tightly localized on the sites which are nearest neighbors of the vacant one, while the $\pi$ electron delocalizes on one of the two sublattices (similarly to the case of several monovalent chemisorbed species such as $\mathrm{H}$ and $\mathrm{F}$ ) as a consequence of the aromatic character of the substrate [19. As a result, the bare vacancy is three-fold symmetric in a doubly-degenerate electronic state and undergoes a Jahn-Teller (JT) distortion, where two of the three carbon atoms above close a pentagon by forming a weak $\mathrm{CC}$

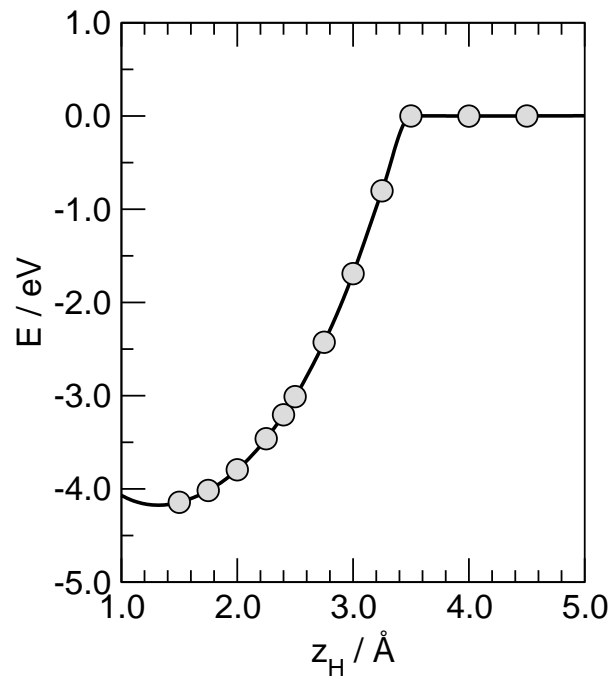

Figure 1: Adsorption energy profile for a single hydrogen atom approaching the vacancy as a function of its height above the surface.

bond [20 26], which may be referred to as JT-induced C$\mathrm{C}$ bond. There are three such possibilities which differ in the identity of the carbon atom opposing the pentagon, the so-called apical atom. The remaining unpaired electrons give rise to singlet and triplet manifolds whose relative stability mainly depends on the height of such apical carbon atom above the surface 26], the planar triplet being most stable. Within the present theoretical approach the electronic ground state has 'dirty' magnetization $M=1.56 \mu_{B}$ because of both the allowance of fractional occupation and the spin-polarized framework (see discussion on this point on Ref. 26), but has an energy which is only few $\mathrm{meV}$ below than that of the correct $M=2 \mu_{B}$ state. Thus, in the following we use this state as a reference state for the bare vacancy.

Adsorption of a first $\mathrm{H}$ atom is strongly exothermic, $\Delta E_{1}=-4.24 \mathrm{eV}$, and barrierless, as shown in Fig 1 where we plot the adsorption profile obtained when optimizing all the atom coordinates but the height of the hydrogen atom above the surface. The $\mathrm{H}$ atom binds to the apical $\mathrm{C}$ atom where much of the unpaired electron density resides, and a strongly covalent bond forms between the two (notice for comparison that a typical value for the $\mathrm{CH}$ binding energy is $\sim 4 \mathrm{eV}$ ). The carbon atom moves slightly out of the plane (and correspondingly the $\mathrm{CH}$ bond gets tilted), suggesting that full $s p^{2}-s p^{3}$ rehybridization of its valence orbitals occurs. This structure has one unpaired electron left, even though the computed magnetization $\left(M=0.56 \mu_{B}\right)$ is lower than one, for the same reasons mentioned above for the bare vacancy; indeed, the energy obtained upon constraining the magnetization to $M=1 \mu_{B}$ is only few meV higher.

When adsorbing a second $\mathrm{H}$ atom at the vacancy site a more complex scenario arises since the two adsorbates may bind to either the same $\mathrm{C}$ atom (geminal configuration) or two distinct $\mathrm{C}$ sites (dimer configuration). 


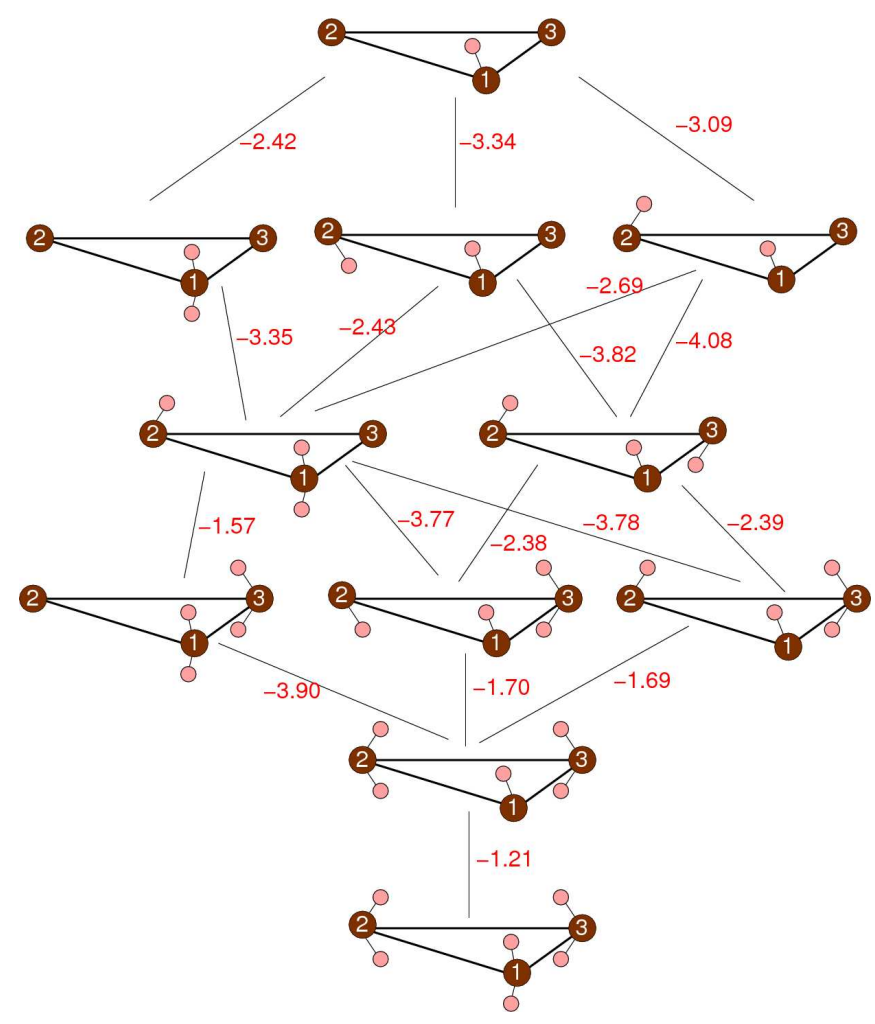

Figure 2: Detailed map of the energetics of the hydrogenated vacancy, showing a schematics of the optimized structures, with the energies $\Delta \Delta E_{n}$ between them.

In the latter case, the two atoms may share the same $($ syn-) or the opposite (anti-) graphene face. All these processes are exothermic when referenced to $\mathrm{H}$ atoms [much more than $\mathrm{H}$ atom adsorption on the basal plane of graphene/graphite, $\Delta E=-0.97 \mathrm{eV}$ according to the most recent works 40] but with a clear preference towards formation of an anti-dimer: $\Delta \Delta E_{2}=-2.42,-3.09$ and $-3.34 \mathrm{eV}$ for the geminal, the syn- and anti-dimer respectively. Activation barriers are small and probably beyond the accuracy limits of the adopted computational approach, but suggestive of a fast adsorption kinetics: they are $22 \mathrm{meV}$ for the geminal product and $21 \mathrm{meV}$ for both dimers.

The above doubly-hydrogenated structures have different magnetic properties, with a net magnetization $M=2.00 \mu_{B}$ for the anti- dimer and $M=0.00 \mu_{B}$ for the geminal one (the syn- structure turns out to have $M=0.00 \mu_{B}$ with two unpaired electrons, and is thus similar in many respects to the anti- isomer). This follows from the different binding processes involved. The first case corresponds overall to the addition of two $\mathrm{H}$ atoms on the weak JT C-C bond of the reconstructed vacancy, a process which breaks such $\mathrm{C}-\mathrm{C}$ bond but leaves the two unpaired electrons of the bare vacancy unaltered. On the other hand, the geminal product may be thought as obtained by double addition to the apical $\mathrm{C}$ atom and corresponding saturation of its unpaired electron density. This is confirmed by the geometrical parameters of the optimized structures: the shortest $\mathrm{C}-\mathrm{C}$ bond of the bare vacancy $\left(d_{C C}=1.98 \AA\right.$; for comparison the remaining two bonds are $2.72 \AA$ long) is almost unaffected in the geminal adduct $\left(d_{C C}=2.06,2.58,2.58 \AA\right)$ while is largely increased (above the value of the longer bonds) in the dimer structures, $d_{C C}=2.82,2.72,2.72 \AA$ and $d_{C C}=2.80,2.65,2.65 \AA$ for the anti- and syn-structures respectively. Notice also that, among the latter, the synstructure experiences an unfavorable $\mathrm{H}-\mathrm{H}$ steric interaction which forces the system to additional relaxation and reduces its stability compared to the anti- one by $\sim 23$ $\mathrm{meV}$.

Next, we consider adsorption of further hydrogen atoms to the three carbon atoms surrounding the vacancy, and their relative arrangements, see the schematics reported in Fig. 2 as a reference. Similarly to the sticking of the second $\mathrm{H}$ atom, three hydrogen atoms may have a geminal pair or sit on different sites. In the latter case they are considered as syn- when they all sit on the same face of the graphene sheet, and anti- otherwise. With four $\mathrm{H}$ atoms formation of a geminal pair is unavoidable, yet the arrangement of the remaining two atoms may be geminal or syn-/anti- dimer. The five and six atom cases parallel in a complementary way the single $\mathrm{H}$ and bare vacancy cases with just one possible arrangement.

The main results of this study can be summarized as follows. Among the three-atom adducts the anti- 
structure is $\sim 1.4 \mathrm{eV}$ most stable than the geminal one $\left(\Delta \Delta E_{3}=-3.82 \mathrm{eV}\right.$ vs. $\left.\Delta \Delta E_{3}=-2.43 \mathrm{eV}\right)$ and the synstructure is not binding at all. The two structures have one unpaired electron (we obtained $M=1.00 \mu_{B}$ in both cases) either because the additional $\mathrm{H}$ atom breaks the JT C-C bond and leaves one unpaired electrons (if we start from the doubly hydrogenated geminal structure and form the $\mathrm{VH}_{3}$ geminal one) or because it couples (at low spin) with one of the two unpaired electrons of the anti-dimer structure and form either the geminal or the anti- $\mathrm{VH}_{3}$ structure. In any case, the JT C-C bond no longer exists and the $\mathrm{C}-\mathrm{C}$ distances are considerably increased $\left(d_{C C}=2.75,2.72,2.62 \AA\right.$ in the geminal and $d_{C C}=2.86,2.85,2.81 \AA$ in the anti- structure). The energetics follows consistently: starting from the most stable anti-dimer structure addition of a third $\mathrm{H}$ atom is easier (and more exothermic) when a $\sigma$ bond is formed on the apical atom than when $H$ binds to an already hydrogenated site to form a geminal product, since in the latter case a bond is formed with the $\pi$-like electron left unpaired on that site.

Among the possible four-fold hydrogenated structures the syn- is slightly more stable than the anti- $\left(\Delta \Delta E_{4}=\right.$ $-2.39 \mathrm{eV}$ vs. $\Delta \Delta E_{3}=-2.38 \mathrm{eV}$ ) and definitely more stable than the geminal one $\left(\Delta \Delta E_{4}=-1.57 \mathrm{eV}\right.$ starting from the geminal $\mathrm{VH}_{3}$ structure). At this stage all the four unpaired electrons initially available upon vacancy formation get saturated with hydrogen, and both the syn- and the anti-structures are non-magnetic, differently from the geminal one which has two unpaired electrons (we obtained $M=0.00 \mu_{B}$ in the first two cases and $M=2.00 \mu_{B}$ in the latter). Further hydrogenation is remarkably less exothermic $\left(\Delta \Delta E_{5}=-1.69 \mathrm{eV}\right.$ and $\Delta \Delta E_{6}=-1.21 \mathrm{eV}$ ) since it necessarily requires further breaking of the aromaticity, and produces magnetic structures with one and two unpaired electrons, respectively. Indeed, compared to the bare vacancy, full hydrogenation requires (i) saturation of the three $\sigma$ dangling bonds, (ii) saturation of the $\pi$ (midgap) state and (iii) breaking of two $\mathrm{C}-\mathrm{C}$ double bonds (a first $\mathrm{C}-\mathrm{C}$ double bond needs to be broken when hydrogenating the $\mathrm{VH}_{4}$ anti- or syn- structure).

Fig. 3 gives an overview of the energetics of the hydrogenated carbon atom vacancy in graphene. There, the H-atom binding energies (or affinities) $D_{n}=-\Delta \Delta E_{n}$ to form the most stable $n$-times hydrogenated structure from the most stable $n-1$ isomer are plotted as a function of $n$. Such structures are the anti- structures, the $n=4$ case being an exception which shows a slight $(\sim 0.01$ $\mathrm{eV}$ ) preference towards the syn- structure. For comparison, the values of the $\mathrm{H}$ binding energy in several different situations are given as horizontal lines; such values comprise the adsorption energy to the basal graphene plane 40, the binding energy of the secondary $\mathrm{H}$ atom to form a para- dimer[19] (a similar result holds for the orto- dimer 19]) and the binding energy for adsorption on an armchair edge and a zig-zag edge which are $\mathrm{H}$ terminated 41]. The latter represents the largest energy

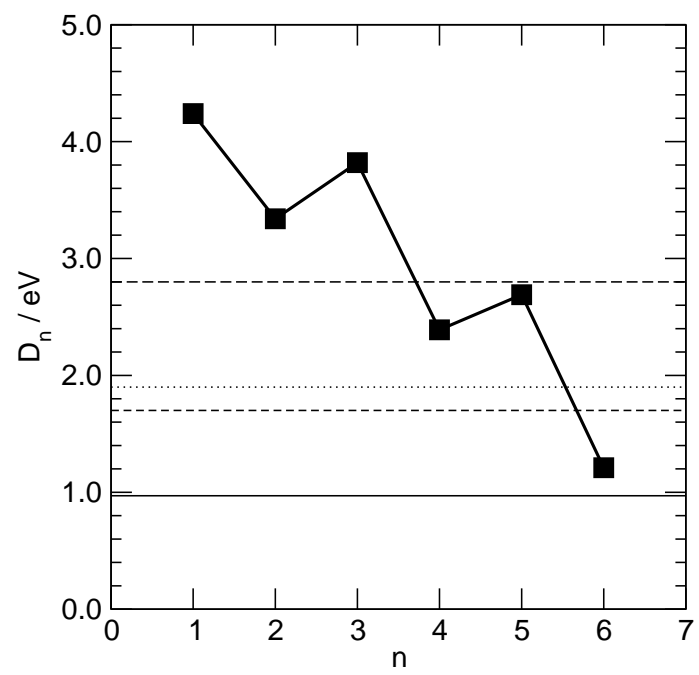

Figure 3: $\mathrm{H}$ atom binding energies for the most stable $V H_{n}$ structures. Horizontal lines are reference values for the $\mathrm{H}$ binding energy in the 'bulk' (full line), for the secondary $\mathrm{H}$ adsorption to form para- dimers (dotted line), and for adsorption at an armchair (dashed) or zig-zag (long-dashed) edge.

gain when adsorbing $\mathrm{H}$ on pristine graphene and compare rather well with the binding energies of the fourth and fifth hydrogen atoms; larger energies are obtained for the first three $\mathrm{H}$ atoms where bonding involves $\sigma$ orbitals and/or unpaired electrons.

Of interest are also some general structural changes accompanying hydrogenation. We find that structures with $n \geq 4$ are more open than the less hydrogenated ones, with $d_{C C} \gtrsim 2.7 \AA$. This value has to be compared with the corresponding value in graphene (i.e. the nextto-nearest neighbors $\mathrm{C}-\mathrm{C}$ distance in the bare, unrelaxed vacancy) which is $d_{C C}=2.46 \AA$. This 'expansion' is accompanied by a substantial movement of the $\mathrm{C}$ atoms out of the plane, up to $0.7-0.8 \AA$. For instance, in the $\mathrm{VH}_{3}$ anti- structure we obtained $\delta z_{C}=0.68,049$ and $-0.34 \AA$ and in the $\mathrm{VH}_{5}$ structure $\delta z_{C}=0.79,0.66$ and $0.18 \AA$. C-H bond lengths fall in the range $1.07-1.12$ $\AA$, bonds in geminal pairs being the longest and almost similar to $\mathrm{CH}$ in perfect graphene $(1.13 \AA)$. With the same token, $\mathrm{H}-\mathrm{H}$ distances in geminal pairs all fall in the range $d_{H H}=1.73-1.79 \AA$ and distances in syn- and anti- pairs are generally larger than these values, but smaller values are possible for syn- pairs. For instance we found $d_{H H}=1.51 \AA$ for the $\mathrm{VH}_{2}$ syn- structure and $d_{H H}=1.42 \AA$ for the syn- pair in the $\mathrm{VH}_{3}$ anti- structure.

\section{B. Thermodynamic analysis}

With the whole energetics of the hydrogenated vacancy at hand we performed a thermodynamic analysis to investigate the relative stability of the various hydrogenated 


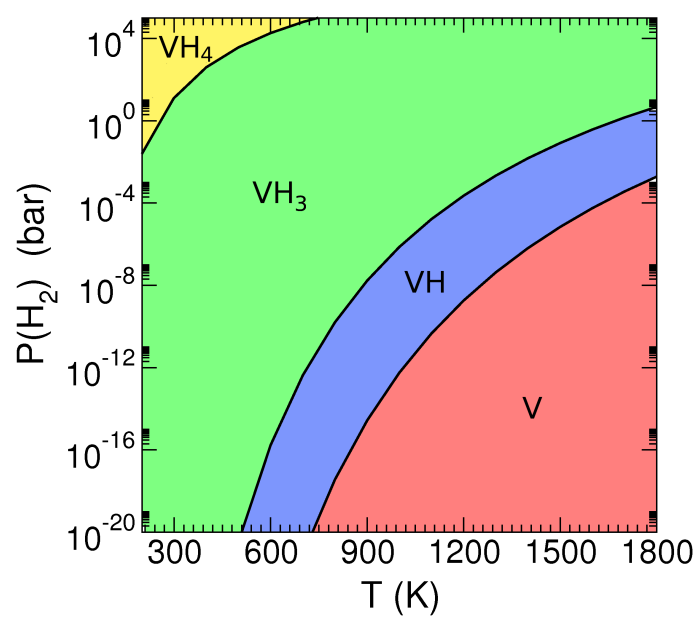

Figure 4: Phase diagram for the hydrogenated C-vacancy in graphene, showing the most stable hydrogenated species $V H_{n}$.

species under reasonable hydrogen partial-pressure and temperature conditions. We considered the lowest molar Gibbs free energy of formation $\Delta G_{n}^{f}(p, T)$ at a $\mathrm{H}_{2}$ partial pressure $p$ and temperature $T$, which -analogously to Eq. (1) - is given by

$$
\Delta G_{n}^{f}(p, T)=G_{V H_{n}}(p, T)-G_{V}(p, T)-\frac{n}{2} G_{H_{2}}(p, T)
$$

where $G_{V H_{n}}, G_{V}$ and $G_{H_{2}}$ are Gibbs free energies for the $n$-times hydrogenated vacancy, the bare vacancy and molecular hydrogen, respectively. For the first two terms we neglected any temperature and pressure dependence and thus relied on the DFT energies above $\left(G_{V H_{n}} \simeq E_{V H_{n}}\right.$ for $n=0,1$, etc.). For hydrogen, some thermodynamic data are available from standard tables 42 and ideal-gas dependence on its partial pressure $p$ can be assumed to write

$$
\begin{aligned}
G_{H_{2}}(p, T) & =H_{H_{2}}\left(p^{\ominus}, T\right)-T S_{H_{2}}\left(p^{\ominus}, T\right)+ \\
& +R T \ln \left(\frac{p}{p^{\ominus}}\right) \equiv E_{H_{2}}+\Delta G_{H_{2}}(p, T)
\end{aligned}
$$

where $R$, as usual, is the perfect gas constant, $S_{H_{2}}\left(p^{\ominus}, T\right)$ is the hydrogen molar entropy at temperature $T$ and standard pressure $p^{\ominus}=1$ bar, and the enthalpy is written as $H_{H_{2}}=E_{H_{2}}+\frac{5}{2} R T$ for $T<T^{\ominus}$ and $H_{H_{2}}=$ $E_{H_{2}}+\frac{5}{2} R T^{\ominus}+\Delta H_{H_{2}}\left(p^{\ominus}, T\right)$ otherwise. Here $E_{H_{2}}$ is the DFT energy of $\mathrm{H}_{2}$ in its equilibrium configuration and $\Delta H_{H_{2}}\left(p^{\ominus}, T\right)$ is the $\mathrm{H}_{2}$ molar enthalpy change from $T=T^{\ominus}=298.15 \mathrm{~K}$ to $T$ at the standard pressure $p^{\ominus}$ (Ref. 42). $\Delta G_{H_{2}}(p, T)$ is defined by the above equation and is the appropriate free-energy change from $T=0$ to $T, p$. Accordingly,

$$
\Delta \Delta G_{n}=\Delta G_{n}^{f}-\Delta G_{n-1}^{f}=\Delta \Delta E_{n}^{m}-\frac{1}{2} \Delta G_{H_{2}}(p, T)
$$

where $\Delta \Delta E_{n}^{m}$ differs from the energy for sequential adsorption defined above by half the dissociation energy
$D_{m}$ of the $H_{2}$ molecule,

$$
\Delta \Delta E_{n}^{m}=E_{V H_{n}}-E_{V H_{n-1}}-\frac{1}{2} E_{H_{2}}=\Delta \Delta E_{n}+\frac{1}{2} D_{m}
$$

where $D_{m}=4.5 \mathrm{eV}$ with our setup.

The results of such an analysis are reported in Fig. 4 in the temperature range $T=250-1800 \mathrm{~K}$ and for a wide hydrogen partial pressure range $p=10^{-20}-10^{5}$ bar, comprising standard atmospheric conditions $(T \sim 300 \mathrm{~K}$ and $p=5.55 \times 10^{-7}$ bar in dry atmosphere) and ultra high vacuum conditions (UHV) where the pressure is $\sim 10^{-12}$ times smaller than the standard value; $p=1$ bar and higher, on the other hand, can be achieved upon hydrogen exposure. Fig. 4 shows that the bare vacancy is thermodynamically stable only at high temperature and low hydrogen pressure; for instance, thermal annealing at $T>1200 \mathrm{~K}$ would be required in atmospheric conditions to free vacancy defects from hydrogen atoms, whereas annealing at $T=600 \mathrm{~K}$, while sufficient for desorbing $\mathrm{H}$ atoms and dimers from the basal plane 43, is not enough to desorb $\mathrm{H}$ atoms form the vacancy. Even under UHV conditions annealing at $T \gtrsim 800 \mathrm{~K}$ is required to have bare vacancies, thereby suggesting that, at least for naturally occurring vacancies, hydrogen passivation is ubiquitary and extensive, with the triply hydrogenated species most abundant up to $T \sim 600 \mathrm{~K}$. Different results may be expected when vacancies are intentionally introduced into the substrate by e.g. electron or ion bombardment, since the hydrogenation kinetics and ensuing equilibration may be considerably slowed down by the low operating partial pressures and temperatures. For instance, STM experiments are often performed at low temperatures $(T \sim 10 \mathrm{~K})$ where a $\mathrm{VH}_{3}$ isomer is the most stable species under a wide range of pressures, but where the hydrogenation kinetics is extremely slow unless high hydrogen partial pressures are used. Similarly for TEM experiments, despite the larger temperature typically used (ambient conditions).

Noteworthy is the absence in Fig. 4 of any doubly hydrogenated species, neither the geminal nor the syn/anti- dimer: upon increasing the temperature at constant pressure the most stable isomers change from the triply hydrogenated form to the singly hydrogenated one, without passing from a $(p, T)$ region where dimers are stable against hydrogenation/dehydrogenation; see also Fig. 5 which reports the molar free-energies of the most stable isomers for different temperatures, at ambient hydrogen partial pressure.

Finally, it is worth noticing that, as a consequence of the energetics discussed in the previous subsection, no geminal species prevails at equilibrium. Geminal adducts have been recently invoked to explain long-time oscillations in the zero-field $\mu$-SR decay asymmetry 44, 45, which were attributed to dipolar interactions between a muonion $(\mathrm{Mu}$, a $\mathrm{H}$ isotope $\sim 10$ times lighter than $\mathrm{H}$ ) and a $\mathrm{H}$ atom in a $-\mathrm{CHMu}$ geminal structure. Though not stable at the experimental conditions $(T=300 \mathrm{~K})$ these structures might easily form from the stable singly- 


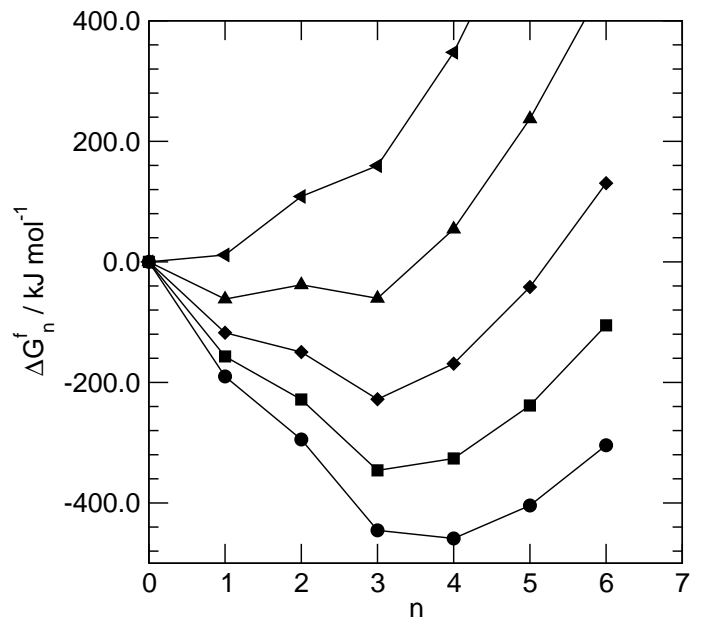

Figure 5: Molar free-energy of formation for the most stable isomers $V H_{n}$ at atmospheric conditions $p=0.55 \times 10^{-6}$ bar, for different temperatures. Circles, squares, rhombus, triangles up and triangles down for $T=0,300,600,1000$ and 1500 $\mathrm{K}$, respectively.

and triply- hydrogenated species prevailing under a wide range of conditions, and dominate the $\mu$-SR signal over alternative arrangements because of structural reasons. Among these, as observed above, the $\mathrm{H}-\mathrm{H}$ distance in geminals has a well defined value $\left(d_{H H} \simeq 1.75 \AA\right.$, in good agreement with the dipolar distance extracted by the $\mu$-SR signal, $d=1.70 \pm 0.02 \AA$ ), irrespective of the specific structure and unaffected by low-frequency vibrations of the lattice (the lowest-frequency motion altering such distance is a H-C-H bending). However, further investigation is required to establish whether such a product can indeed form in a collision between a muonion and a hydrogenated vacancy.

\section{Magnetic properties}

The magnetic properties of carbon atom vacancies in graphene have been recently subjected to experimental investigations with conflicting results. Early report of paramagnetism due to spin-1 species 46, in accordance with a triplet ground-state configuration of the bare vacancy [26], have been questioned on the light of magnetometry measurements on carefully prepared samples, which showed the presence of spin- $1 / 2$ species for both flourine ad-species and vacancies [16], at odds with the different numbers of unpaired electrons left upon defect formation. Later measurements from the same group showed that in the presence of carbon atom vacancies two different spin-1/2 local magnetic moments contribute to the observed paramagnetic signal, one arising from the $\sigma$ network and one from the $\pi$ band system[17]. This finding would agree with the above mentioned electronic structure of the Jahn-Teller distorted vacancy if the singlet-triplet separation (Hund's coupling) were van-

\begin{tabular}{|l|c|c|c|}
\hline & $M / \mu_{B}$ & $m$ & Character \\
\hline \hline $1 \mathrm{H}$ & 0.56 & 1 & $\pi$ \\
\hline 2H geminal & 0.00 & 0 & - \\
\hline 2H anti & 2.00 & 2 & $\sigma, \pi$ \\
\hline 2H syn & 0.00 & 2 & $\sigma, \pi$ \\
\hline 3H geminal & 1.00 & 1 & $\sigma$ \\
\hline 3H anti & 1.00 & 1 & $\pi$ \\
\hline 4H geminal & 1.92 & 2 & $\sigma, \pi$ \\
\hline 4H anti & 0.00 & 0 & - \\
\hline 4H syn & 0.00 & 0 & - \\
\hline $5 \mathrm{H}$ & 1.00 & 1 & $\pi$ \\
\hline $6 \mathrm{H}$ & 2.00 & 2 & $\pi, \pi$ \\
\hline
\end{tabular}

Table I: Computed total magnetization $M$ in $\mu_{B}$ and predicted number $m$ of unpaired electrons left on the C-vacancy after the hydrogenation process, along with their character.

ishing small to allow a decoupled response of the two unpaired electrons left upon reconstruction, a possibility which has been recently ruled out by accurate $a b$ initio results 26]. In the same work it has been shown that the energetics is sensitive to the out-of-plane motion of the apical carbon atom, thereby suggesting that ripples and/or interactions with a substrates could explain why the above mentioned unpaired electrons of the vacancy are uncoupled. Here we consider a different scenario, i.e. the possibility that the vacancy is hydrogenated to some extent and thus presents a net magnetic moment which depends on the degree of hydrogenation.

Table II summarizes the computed magnetic moment for each structure investigated, along with the number of unpaired electrons predicted with the help of the resonating valence bond model, and their 'character', i.e. whether they are $\sigma$ or $\pi$ and thus localized on a single lattice position or delocalized over several lattice sites. Notice though that " $\pi$-moments" entries in the Table are most often hybrid $\sigma-\pi$ moments: for instance, the singly hydrogenated structure can be thought as originated from binding a $\mathrm{H}$ atom to one of the two $s p^{3}$ hybrid orbitals of the apical carbon atom of the bare vacancy, a process which leaves one unpaired electron in a $s p^{3}$ orbital free to hybridize with $p_{Z}$ states of neighboring $\mathrm{C}$ atoms and form $s p^{3}-\pi$ states.

With only few exceptions the computed magnetic moments refer to pure spin states and thus agree well with the number of unpaired electrons predicted by the resonance structures. For instance -as already mentioned above- among the doubly hydrogenated structures, the geminal one has no magnetic moment since it results from saturating both dangling bonds on the apical $\mathrm{C}$ atom of the bare vacancy, while the anti- structure has the same magnetic moment as the bare vacancy because the two $\mathrm{H}$ atoms bind to the JT bond; interestingly, in synisomer, the singlet-triplet ordering seems to be reversed and a net zero megnetic moment with two unpaired electrons signals an open-shell singlet. 
A quick look at Table $\llbracket$ reveals that hydrogenated vacancies have magnetic moments in the range $0.0-2.0 \mu_{B}$, even though the corresponding singly occupied states may have very different character. For instance, despite the presence of one unpaired electron in both the $\mathrm{VH}_{3}$ geminal and the $\mathrm{VH}_{3}$-anti structure, the corresponding singly occupied state has either a $\sigma$ or a $\pi$ origin. More interestingly, taking into account the results of the previous section, we observe that in a wide range of temperature and hydrogen partial pressure of interest in many practical situations, the magnetic moment of the most stable hydrogenated species $\left(\mathrm{VH}_{1}, \mathrm{VH}_{3}\right.$ and $\mathrm{VH}_{4}$ structures $)$ has one and the same value, namely $M=1.00 \mu_{B}$. Thus, with the exception of the bare vacancies, thermodynamically stable vacant species on a wide range of ambient conditions are expected to behave as spin- $1 / 2$ magnetic moments as a consequence of hydrogenation.

\section{STM imaging}

Finally, in order to help identifying and distinguishing the various hydrogenated species investigated we performed, on a few relevant optimized structures, simulations of the STM images within the standard TersoffHamann approximation. The sample bias was fixed at $-0.5 \mathrm{~V}$ and constant-current images obtained by integrating the local density of states in the range $[-0.5,0]$ $\mathrm{eV}$ from the Fermi level, as the (largest) height $z$ of an isosurface of the resulting function.

Several situations were envisaged. First we considered the bare vacancy which, in its stable structure, should present a distorted configuration, with a C-C distance sensibly shorter than the other two. However, since three equivalent minima are possible, each differing by the orientation of the JT bond, we also considered the possibility that orientational averaging occurs on the time-scale of imaging (few seconds). This could happen either because of thermal hopping between the minima, or because a dynamic Jahn-Teller effect is operative whereby the ground-state structure is a superposition of the three possible distorted one and robust against environmental dephasing. Fig. 6 shows these two possibilities, panel (a) for the averaged structure and panel (b) for the distorted one, the latter with a superimposed molecular model to help identifying the lattice positions. As is evident from Fig.s 6(a,b), despite the structural differences, the distorted and the symmetrical configurations of the bare vacancy are hardly distinguishable from each other. Both structures present a bright signal with exact or approximate three-fold symmetry which extends far from the vacant site and which corresponds to the above mentioned $\pi$-midgap state, in agreement with STM/STS experiments [10], but no further distinguishing feature.

A distorted structure similar to that of the bare vacancy but with a locked configuration appears upon single hydrogenation. In this case, the attached $\mathrm{H}$ atom is not free to move on neighboring $\mathrm{C}$ atoms of the va- cancy (even though we did not compute it, the barrier for hopping likely matches the desorption barrier, as happens for hydrogen atoms adsorbed on the basal plane of graphene 18, 19]) and the simulated STM image, reported in Fig. 6 (c), shows a clearer breaking of symmetry than in the case of the bare vacancy. It further shows an increase of brightness in the vacancy at the expense of the a reduced intensity of the signal due to the $\pi$ - midgap state, probably related to the admixture of $\sigma$ and $\pi$ character (remember that the $\mathrm{H}$ atom is accommodated out-of-plane with a $\mathrm{CH}$ bond originating from a $s p^{3} \mathrm{C}$ orbital, and an unpaired electron is left on the remaining $s p^{3}$ orbital, free to hybridize with the $\pi$-band states. Fig. 6 is the simulated image for the structure with the $\mathrm{H}$ atom above the plane).

Finally, we considered one further thermodynamically stable structure, the triply hydrogenated anti-structure which prevails under standard conditions. Here, too, a number of equivalent configurations is possible (three possible orientations of the dimers in syn-relationships times two possible positions, above or below the surface plane) and we decided to average over them since their interconversion does not involve any $\mathrm{H}$ atom transfer and should thus be possible under ordinary temperature conditions. Fig. 7 reports the simulated image of the structure which shows a clear increase of the intensity in the vacant site, as well as the expansion of the lattice accompanying hydrogenation which was mentioned above. Note that this structure still bears one unpaired $\pi$-moment of the kind similar to the one in Fig.s 6 (a-c) and thus, far from the vacant site, displays the same 'typical' three-fold symmetric signal, though with a reduced intensity.

A symmetric structure has been recently observed by Robertson and co-workers 32 with TEM, under conditions where the vacancy would be triply hydrogenated if equilibrium conditions prevailed. However, our computed C-C distances in the $\mathrm{VH}_{3}$-anti structure $\left(d_{C C} \sim\right.$ $2.8 \AA$ ) are sensibly larger than those found in the experiment $\left(d_{C C}=2.5 \pm 0.1 \AA\right)$ that the observed structure is likely to be a bare vacancy which dynamically switches between its equivalent configurations (or an excited state of the vacancy with electronic different configuration).

\section{CONCLUSIONS}

We reported on a thorough investigation of the structure and energetics of several hydrogenated states of a carbon atom vacancy in graphene. We found large Hatom adsorption energies for the first few hydrogen atoms (related to saturation of $\sigma$-dangling bonds) which progressively decrease towards values similar to those found for adsorption at graphene edges. As a consequence, under equilibrium conditions, triply- and singly- hydrogenated species prevail over a wide range of hydrogen partial pressure and temperatures relevant in many experimental situations. The computed energetics shows 

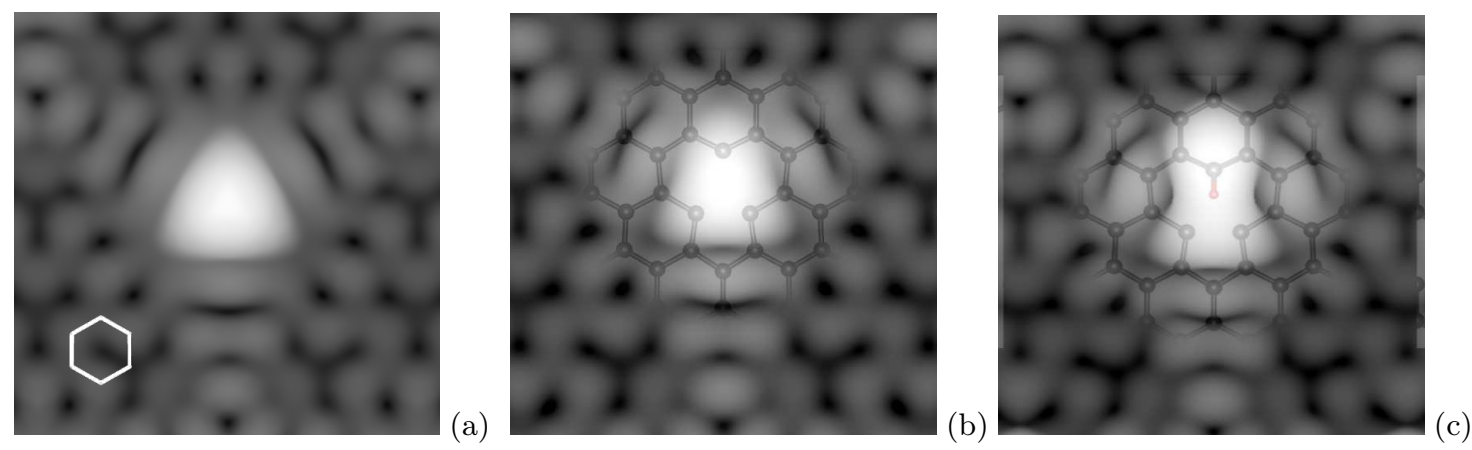

Figure 6: Simulated STM images of the vacancy of the bare vacancy (a,b) and of the singly hydrogenated vacancy (c) with molecular models showing the lattice arrangement. Image on panel (a) has been obtained by averaging over the three equivalent orientations of the structure.

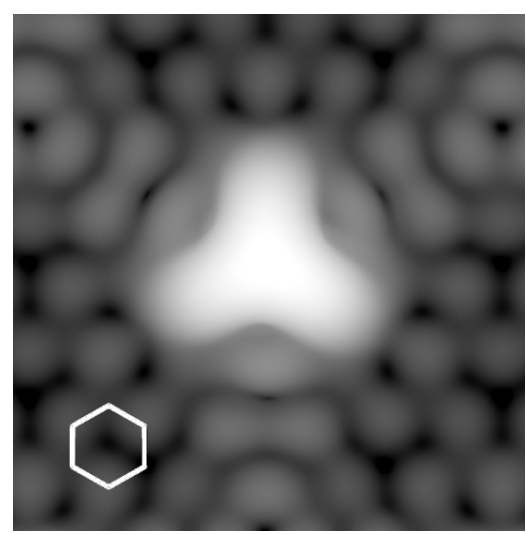

Figure 7: Simulated STM images of the most stable triply hydrogenated isomer of the vacancy, averaged over its equivalent configurations.

that geminal pairs are invariably less stable than different hydrogen arrangements with adsorbed $\mathrm{H}$ atom on different sites (if possible), preferably in an anti- configuration. Thus, whether geminal products are formed (as suggested by recent $\mu$-SR experiments [44, 45]) requires further investigation of the dynamical aspect of the process.

Most of the investigated structures have a net non- zero magnetic moment capable of a paramagnetic signal. In particular, one spin- $1 / 2$ moment related to an unpaired electron in a semilocalized $\pi$-midgap state appears both in the singly- and triply- hydrogenated species which dominate the $(p, T)$ phase-diagram describing hydrogenation.

Comparison with existing STM investigations of defective graphene[10] showed agreement for the presence of the signal due to the $\pi$-midgap state, but further studies are needed to establish whether the observed vacancies are Jahn-Teller distorted, dynamically averaged or hydrogenated to some extent. Simulated STM images hardly distinguish a locked from an averaged bare vacancy but present a few marked features accompanying hydrogenation which may help identifying hydrogenated species.

\section{ACKNOWLEDGMENTS}

The authors acknowledge Quentin Ramasse for useful discussions. This work has been supported by Regione Lombardia and CILEA consortium through an ISCRA and LISA (Laboratory for Interdisciplinary Advanced Simulation) 2012 initiative grants.
[1] A. V. Krasheninnikov and K. Nordlund, J. Appl. Phys. 107, 071301 (2010).

[2] Q. M. Ramasse, R. Zan, U. Bangert, D. W. Boukhvalov, Y. W. Son, and K. S. Novoselov, ACS Nano 6, 4063 (2012).

[3] T. J. Booth, F. Pizzocchero, H. Andersen, T. W. Hansen, J. B. Wagner, J. R. Jinschek, R. E. Dunin-Borkowski, O. Hansen, and P. Bøggild, Nano. Lett. 11, 2689 (2011).

[4] N. Severin, S. Kirstein, I. M. Sokolov, and J. P. Rabe, Nano. Lett. 9, 457 (2009).

[5] M. Bonfanti, S. Casolo, G. Tantardini, A. Ponti, and R. Martinazzo, J. Chem. Phys. 135, 164701 (2011).

[6] H. Terrones, R. Lv, M. Terrones, and M. S. Dresselhaus,
Rep. Prog. Phys. 75, 062501 (2012).

[7] Q. M. Ramasse, C. R. Seabourne, D. M. Kepaptsoglou, R. Zan, U. Bangert, and A. J. Scott, Nano. Lett. 13, 4989 (2013).

[8] V. M. Pereira, J. M. B. Lopes dos Santos, and A. H. Castro Neto, Phys. Rev. B 77, 115109 (2008).

[9] V. M. Pereira, F. Guinea, J. Lopes dos Santos, N. Peres, and A. Castro Neto, Phys. Rev. Lett. 96, 036801 (2006).

[10] F. G. M. M. Ugeda, I. Brihuega and J. M. GomezRodriguez, Phys. Rev. Lett. 104, 096804 (2010).

[11] N. M. R. Peres, F. Guinea, and A. H. castro Neto, Phys. Rev. B 73, 125411 (2006).

[12] J. P. Robinson, H. Schomerus, L. Oroszlány, and V. I. 
Fal'ko, Phys. Rev. Lett. 101, 196803 (2008).

[13] T. O. Wehling, S. Yuan, A. I. Lichtenstein, A. K. Geim, and M. I. Katsnelson, Phys. Rev. Lett. 105, 056802 (2010).

[14] N. M. R. Peres, Rev. Mod. Phys. 82, 2673 (2010).

[15] A. Ferreira, J.Viana-Gomes, J. Nilsson, E. R. Mucciolo, N. Peres, and A. C. Neto, Phys. Rev. B 75, 165402 (2011).

[16] R. R. Nair, M. Sepioni, I.-L. Tsai, O. Lehtinen, J. Keinonen, A. V. Krasheninnikov, T. Thomson, A. K. Geim, and I. V. Grigorieva, Nature Phys. 8, 199 (2012).

[17] R. R. Nair, I.-L. Tsai, M. Sepioni, O. Lehtinen, J. Keinonen, A. V. Krasheninnikov, A. H. C. Neto, M. I. Katsnelso, A. K. Geim, and I. V. Grigorieva, arXiv:1301.7611 (2013).

[18] L. Hornekær, E. Rauls, W. Xu, Ž. Šljivančanin, R. Otero, I. Stensgaard, E. Læegsgaard, B. Hammer, and F. Besenbacher, Phys. Rev. Lett. 97, 186102 (2006).

[19] S. Casolo, O. M. Løvvik, R. Martinazzo, and G. F. Tantardini, J. Chem. Phys. 130, 054704 (2009).

[20] A. A. El-Barbary, R. H. Telling, C. P. Ewels, M. I. Heggie, and P. R. Briddon, Phys. Rev. B 68, 144107 (2003).

[21] P. O. Lethinen, A. S. Foster, Y. Ma, A. V. Krasheninnikov, and R. M. Nieminen, Phys. Rev. Lett. 93, 187202 (2004).

[22] O. V. Yazyev and L. Helm, Phys. Rev. B 75, 125408 (2007).

[23] M. W. C. Dharma-Wardana and M. Z. Zgierski, Physica E 41, 80 (2008).

[24] X. Q. Dai, J. H. Zhao, M. H. Xie, Y. N. Tang, Y. Li, and B. Zhao, Eur. Phys. J. B 80, 343 (2011).

[25] J. J. Palacios and F. Yndurain, Phys. Rev. B 85, 245443 (2012).

[26] M. Casartelli, S. Casolo, G. F. Tantardini, and R. Martinazzo, Phys. Rev. B 88, 195424 (2013).

[27] H. A. Mizes and J. S. Foster, Science 244, 559 (1989).

[28] J. G. Kushmerick, K. F. Kelly, H.-P. Rust, N. J. Halas, and P. S. Weiss, J. Phys. Chem. B 103, 1619 (1999).

[29] T. Kondo, Y. Honma, J. Oh, T. Machida, and J. Naka- mura, Phys. Rev. B 82, 153414 (2010).

[30] K. F. Kelly, D. Sarkar, G. D. Hale, S. J. Oldenburg, and N. J. Halas, Science 273, 1371 (1996).

[31] Y. Niimi, H. Kambara, T. Matsui, D. Yoshioka, and H. Fukuyama, Phys. Rev. Lett. 97, 236804 (2006).

[32] A. W. Robertson, B. Montanari, K. He, C. S. Allen, Y. A. Wu, N. E. Harrison, A. I. Kirkland, and J. H. Warner, ACS Nano 7, 4495 (2013).

[33] G. Kresse and J. Hafner, Phys. Rev. B 49, 14251 (1994).

[34] G. Kresse and J. Furthmüller, Phys. Rev. B 54, 11169 (1996).

[35] J. P. Perdew, K. Burke, and M. Ernzerhof, Phys. Rev. Lett. 77, 3865 (1996).

[36] J. P. Perdew, K. Burke, and M. Ernzerhof, Phys. Rev. Lett. 78, 1396 (1997).

[37] P. E. Blöchl, Phys. Rev. B 50, 17953 (1994).

[38] G. Kresse and D. Joubert, Phys. Rev. B 59, 1758 (1999).

[39] H. Jonsson, G. Mills, and K. W. Jacobsen, 'Nudged Elastic Band Method for Finding Minimum Energy Paths of Transitions', in 'Classical and Quantum Dynamics in Condensed Phase Simulations' (World Scientific, 1998).

[40] V. V. Ivanovskaya, A. Zobelli, D. Teillet-Billy, N. Rogeau, V. Sidis, and P. R. Briddon, Eur. Phys. J. B 76, 481 (2010).

[41] K. May, B. V. Unterreiner, S. Dapprich, and R. Ahlrichs, Phys. Chem. Chem. Phys. 2, 5089 (2000).

[42] http://webbook.nist.gov, nIST Chemistry Webbook.

[43] L. Hornekær, Ž. Šljivančanin, W. Xu, R. Otero, E. Rauls, I. Stensgaard, E. Lægsgaard, B. Hammer, and F. Besenbacher, Phys. Rev. Lett. 96, 156104 (2006).

[44] M. Riccó, D. Pontiroli, M. Mazzani, M. Choucair, J. A. Stride, and O. V. Yazyev, Nano. Lett. 11, 4919 (2011).

[45] M. Riccó, M. Aramini, M. Mazzani, D. Pontiroli, M. Gaboardi, and O. V. Yazyev, Phys. Scr 88, 068508 (2013)

[46] A. Ney, P. Papakonstantinou, A. Kumar, N.-G. Shang, and N. Peng, Appl. Phys. Lett. 99, 102504 (2011). 